\title{
The unfolded protein response mediates reversible tau phosphorylation induced by metabolic stress
}

\author{
JM van der Harg ${ }^{1}$, A Nölle ${ }^{1}$, R Zwart ${ }^{1}$, AS Boerema ${ }^{2}$, ES van Haastert ${ }^{3}$, AM Strijkstra ${ }^{2}$, JJM Hoozemans ${ }^{3}$ and W Scheper ${ }^{*, 1,4,5}$
}

The unfolded protein response (UPR) is activated in neurodegenerative tauopathies such as Alzheimer's disease (AD) in close connection with early stages of tau pathology. Metabolic disturbances are strongly associated with increased risk for AD and are a potent inducer of the UPR. Here, we demonstrate that metabolic stress induces the phosphorylation of endogenous tau via activation of the UPR. Strikingly, upon restoration of the metabolic homeostasis, not only the levels of the UPR markers pPERK, pIRE1 $\alpha$ and BiP, but also tau phosphorylation are reversed both in cell models as well as in torpor, a physiological hypometabolic model in vivo. Intervention in the UPR using the global UPR inhibitor TUDCA or a specific small-molecule inhibitor of the PERK signaling pathway, inhibits the metabolic stress-induced phosphorylation of tau. These data support a role for UPR-mediated tau phosphorylation as part of an adaptive response to metabolic stress. Failure to restore the metabolic homeostasis will lead to prolonged UPR activation and tau phosphorylation, and may thus contribute to AD pathogenesis. We demonstrate that the UPR is functionally involved in the early stages of tau pathology. Our data indicate that targeting of the UPR may be employed for early intervention in tau-related neurodegenerative diseases.

Cell Death and Disease (2014) 5, e1393; doi:10.1038/cddis.2014.354; published online 28 August 2014

Several neurodegenerative disorders are characterized by abnormal phosphorylation and aggregation of the neuronspecific microtubule-binding protein tau. ${ }^{1}$ These so-called tauopathies include highly prevalent neurodegenerative disorders as Alzheimer's disease (AD) and tau-related frontotemporal dementias, for which at present no effective treatment exists. To develop a disease-modifying intervention strategy, it is pivotal to understand early molecular events that lead to the increased phosphorylation of tau. In pathological situations such as AD, tau is hyperphosphorylated and forms aggregates, but under physiological conditions tau phosphorylation and dephosphorylation are a normal dynamic process. Phosphorylation of tau reduces the binding of tau to microtubules and regulates the function of tau in axonal transport. ${ }^{2}$

It has been hypothesized that initially increased tau phosphorylation is a protective mechanism, which may turn pathological if it is present for a prolonged period. ${ }^{3}$ For example, metabolic stress conditions could lead to increased phosphorylation of tau as part of an adaptive response, by temporarily inhibiting energy-costly axonal transport. In line with this, increased tau phosphorylation is observed in brains of animals in torpor, which is a physiological hypometabolic situation whereby the metabolic rate can be reduce by $90 \% .{ }^{4}$ This is often facilitated by a reduction in body temperature, although this is not an absolute prerequisite. ${ }^{5,6}$ In brains of mammals undergoing torpor, tau is hyperphosphorylated and not aggregated. In this physiological situation, the tau phosphorylation is reversed when the animals return to their normal metabolic state. Various in vivo studies demonstrate that tau phosphorylation is also increased in pathological metabolic stress situations (e.g., diabetes type I and II; treatment with a mitochondrial uncoupler). ${ }^{5,711}$ In this case, the metabolic stress is persistent and tau aggregates are formed. Interestingly, metabolic dysfunction is a prominent risk factor for the development of AD. ${ }^{12}$

Our lab and others have previously shown that the unfolded protein response (UPR), the stress response of the endoplasmic reticulum (ER), is activated in the earliest pathological stages in brains of patients with tauopathies. ${ }^{13-16}$ The ER is strategically positioned to sense and integrate metabolic signals. It is an important site for crucial steps in protein, lipid and glucose metabolism. The UPR is initiated upon disturbance of the homeostasis in the ER via activation of three sensor proteins in the ER membrane: PERK, ATF6 and IRE1. Activation of these sensors transfers stress signals to

\footnotetext{
${ }^{1}$ Department of Genome Analysis, Academic Medical Center, Amsterdam, The Netherlands; ${ }^{2}$ Units of Chronobiology and Molecular Neurobiology, Center for Behaviour and Neurosciences, University of Groningen, Groningen, The Netherlands; ${ }^{3}$ Department of Pathology, Neuroscience Campus Amsterdam, VU University Medical Center, Amsterdam, The Netherlands; ${ }^{4}$ Department of Clinical Genetics and Alzheimer Center, VU University Medical Center, Amsterdam, The Netherlands and ${ }^{5}$ Departments of Functional Genomics and Molecular and Cellular Neuroscience, Center for Neurogenomics and Cognitive Research, Neuroscience Campus Amsterdam, VU University, Amsterdam, The Netherlands

${ }^{*}$ Corresponding author: W Scheper, Molecular Neurodegeneration Group, Departments of Functional Genomics and Molecular and Cellular Neuroscience, Center for Neurogenomics and Cognitive Research, Neuroscience Campus Amsterdam, VU University, W\&N building, room B-439, De Boelelaan 1085, Amsterdam 1081 HV, The Netherlands. Tel: +31 20 5982823; Fax: +31 20 5986929; E-mail: w.scheper@vumc.nl

Abbreviations: A, arousal; AD, Alzheimer's disease; ATF6, activating transcription factor 6; BiP/GRP78, immunoglobulin heavy-chain-binding protein /glucose-related protein 78; CHOP, CCAAT/-enhancer-binding protein homologous protein; 2DG, 2-deoxy glucose; eEF2 $\alpha$, eukaryotic elongation factor 2 alpha; elF2 $\alpha$, eukaryotic initiation factor 2 alpha; ER, endoplasmic reticulum; EU, euthermia; GAPDH, glyceraldehyde-3-phosphate dehydrogenase; IRE1, inositol requiring kinase 1; PERK, double-stranded RNA-activated protein kinase (PKR)-like endoplasmic reticulum kinase; p-tau, phosphorylated tau; TE, early torpor; TL, late torpor; TM, tunicamycin; TUDCA, tauroursodeoxycholic acid; UPR, unfolded protein response

Received 22.4.14; revised 11.7.14; accepted 23.7.14; Edited by D Bano
} 
the cytoplasm and the nucleus, resulting in overall inhibition of protein translation and increased transcription and translation of genes involved in the restoration of ER homeostasis. ${ }^{17}$

In brains of AD patients, the UPR is activated in neurons containing diffusely distributed phosphorylated tau ( $p$-tau), but not in neurons with densely aggregated tau, suggesting that UPR activation occurs before the formation of end-stage tau inclusions, the tangles. ${ }^{15,18}$ This strong correlation between UPR activation and early stages of $p$-tau accumulation is not only found in AD but also in other tauopathies. ${ }^{16}$ Interestingly, the p-tau in torpor brain is reminiscent of $p$-tau present in neurons undergoing UPR activation in brains of tauopathy patients: hyperphosphorylated at the same epitopes and not aggregated. ${ }^{19}$ Moreover, in vitro and in vivo data demonstrate that treatment with thapsigargin, which activates the UPR via disturbance of calcium homeostasis, is accompanied by increased tau phosphorylation, suggesting there may be a direct functional connection between UPR activation and tau phosphorylation. ${ }^{20,21}$ Metabolic stress is an important physiological trigger of the UPR. In this study, we investigated whether UPR activation functionally connects metabolic dysfunction and tau phosphorylation as part of a reversible adaptive response.

\section{Results}

Temporal and spatial correlation of reversible UPR and p-tau in a hypometabolic model. To investigate whether p-tau in the torpid brain is also associated with UPR activation, brains of hamsters in euthermia, early torpor, late torpor, and after arousal were analyzed. Antibodies for phosphorylated PERK (pPERK) and phosphorylated IRE1 $\alpha$ $(p I R E 1 \alpha)$ were used as markers for UPR activation in combination with AT8, a p-tau antibody. In the euthermic state UPR activation and p-tau are not detected, whereas in early and late torpor massive UPR activation and $p$-tau is observed in the hippocampus (Figure 1a) and cortex (Figure 1b). There is no obvious difference visible between early and late torpor. Interestingly, pPERK, pIRE $1 \alpha$ and $p$-tau disappear again completely after arousal when metabolism and temperature are restored (Figures $1 \mathrm{~d}, \mathrm{~h}$ and $\mathrm{l}$ ). Quantification of the images confirms the strong induction of UPR activation and p-tau in early torpor and late torpor (20-80 fold increased relative to euthermia) in the cortex and hippocampus (Figure 2). pPERK and p-tau positivity are not significantly different between early and late torpor and show a similar pattern throughout the hibernation cycle (Figures $2 b$ and c). The pIRE $1 \alpha$ staining shows a decrease in late torpor compared with early torpor in the cortex and hippocampus, but the levels are still 40-60 fold higher than under euthermic conditions (Figure 2a). Interestingly, quantification of the UPR markers and p-tau shows no significant difference between arousal and euthermia, indicating that UPR activation and tau phosphorylation are fully reversible processes, which are only activated during torpor. It has been reported that during torpor tau is phosphorylated at multiple sites; ${ }^{5}$ western blotting of Syrian hamster brain lysates during euthermia, torpor and arousal confirms that also tau phosphorylation at Ser396 is increased during torpor and reversed upon arousal (Supplementary Figure 1).
The pPERK, pIRE1 $\alpha$ and p-tau reactivities show similar distribution between brain areas. In many areas positive cells are observed during torpor, but the intensity and the number of positive cells varies between brain regions; cortex and hippocampus show the most pronounced positivity, whereas the striatum is virtually negative (Figures $2 a-c$; Supplementary Figure 2). To investigate this spatial correlation in more detail, double immunofluorescence was performed and analyzed by confocal microscopy. The immunohistochemistry shows that pPERK and pIRE $1 \alpha$ are present in the cell bodies of neurons but not in neurites or axons, whereas p-tau is detected in neuronal cell bodies, but also in neurites and very prominently in axons (Figure 3). The confocal pictures show that, like in the human AD brain, ${ }^{15}$ pPERK and pIRE $1 \alpha$ are present as punctate structures in the soma, whereas p-tau shows a more diffuse distribution throughout the cell. Interestingly, despite their different subcellular distribution, $>75 \%$ of the neurons display UPR activation as well as $p$-tau (Figures $3 d$ and $h$ ). Isolated p-tau staining is observed in $<14 \%$ and single pPERK or pIRE $1 \alpha$ staining in $<10 \%$ of the neurons. These data demonstrate that UPR activation and tau phosphorylation during torpor occur in the same neurons in specific brain areas. Striking is the reversibility of both UPR activation and tau phosphorylation during arousal when metabolism returns to normal.

The UPR is activated by metabolic stress and consolidated by hypothermia. It is clear that the UPR is activated during torpor; however, it is not known whether this is due to a passive temperature-driven regulation, or that it is actively induced by a torpor-related mechanism. Both pPERK and pIRE1 $\alpha$ that were used as UPR markers in the hamster brain in torpor are activated by phosphorylation. It has been reported that the activity of phosphatases decreases exponentially at temperatures lower than $37^{\circ} \mathrm{C},{ }^{22}$ which can potentially cause a passive increase in the levels of these markers. Therefore, we analyzed the phosphorylation of PERK in an in vitro model. Differentiated human neuronal SK-N-SH cells were incubated at euthermic temperature of $37^{\circ} \mathrm{C}$ and at $26^{\circ} \mathrm{C}$ to investigate UPR activation at decreased temperatures. In addition, the differentiated SK-N-SH cells were treated with two different UPR inducers, tunicamycin (TM) and 2-deoxy glucose (2DG). 2DG inhibits glucose metabolism at the first step of glycolysis and thus blocks ATP production from glucose, similar to the drastic decrease in metabolism that occurs during torpor. The phosphorylation of PERK is readily increased at $37^{\circ} \mathrm{C}$ upon treatment with TM and 2DG (Figure 4), as expected. However, lowering the temperature to $26^{\circ} \mathrm{C}$ does not induce pPERK levels. Moreover, the induction of PERK phosphorylation by TM or $2 D G$ is completely abolished at $26^{\circ} \mathrm{C}$. Because the UPR affects transcription, translation and phosphorylation, we analyzed more UPR targets at mRNA and protein levels at different time points. We obtained similar results for all targets demonstrating that hypothermia does not result in UPR activation (Supplementary Figures 3 and 4). Our data demonstrate that the UPR sensors as well as the downstream signaling of the UPR is not spontaneously activated at lower temperatures and therefore UPR activation is not a 
A
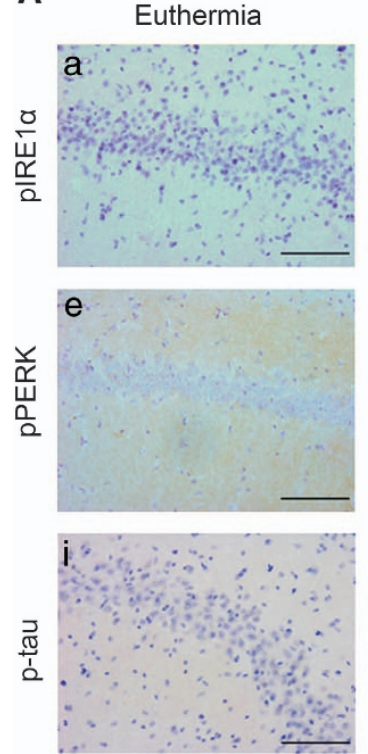

B
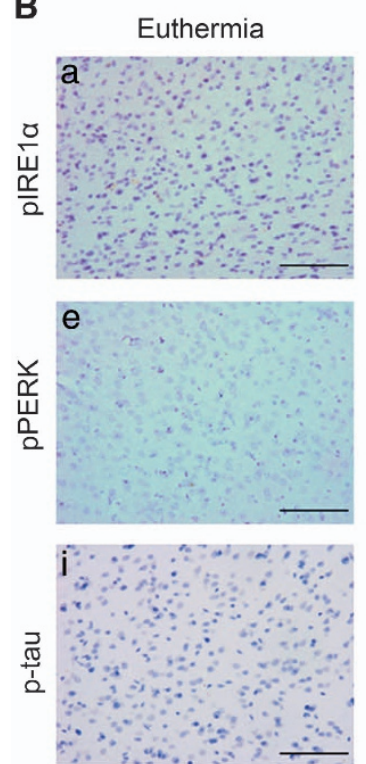

Early Torpor
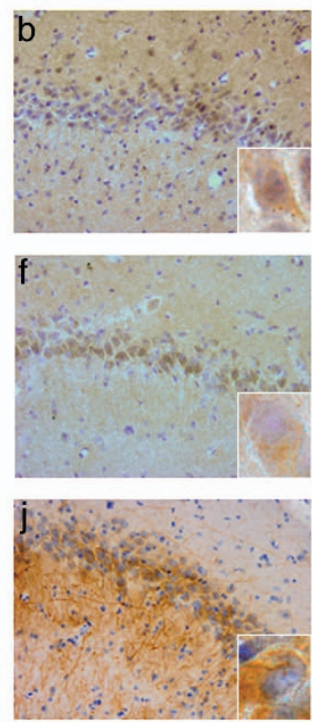

Early Torpor
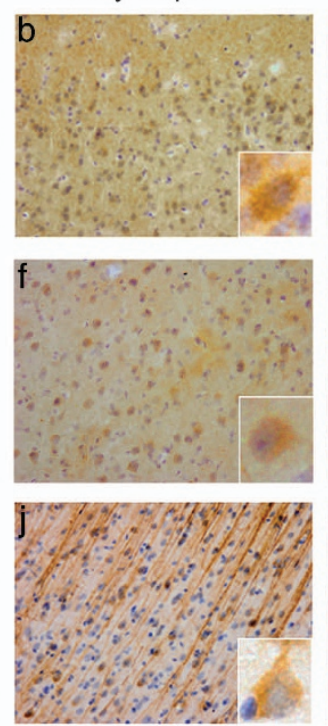

Late Torpor
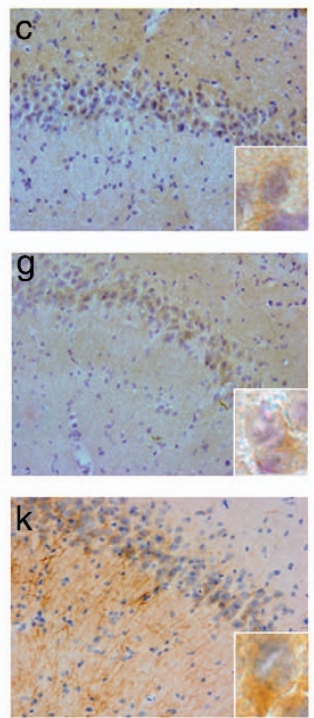

Late Torpor
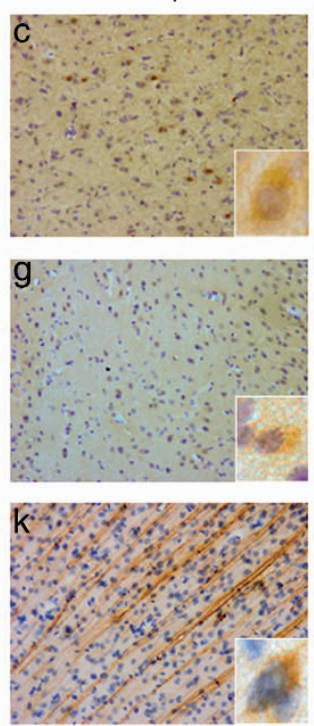
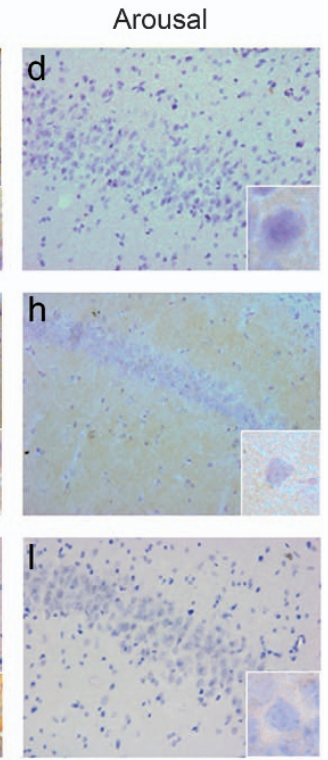

Arousal
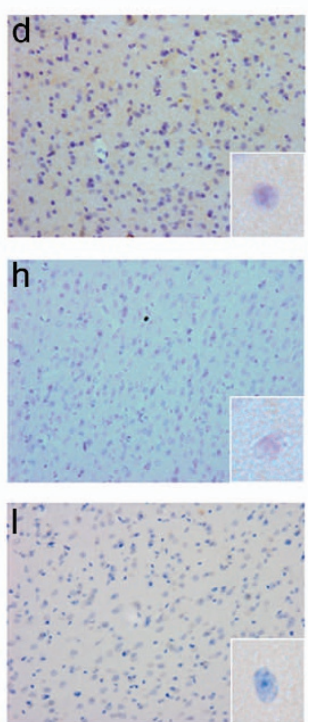

Figure 1 Temporal correlation between UPR activation and p-tau. Coronal brain sections of wild-type Syrian hamsters were studied during euthermia, early torpor, late torpor and arousal. For each condition three to five animals were used for immunohistochemistry. All animals of the same condition showed similar staining patterns. Representative immunohistochemical staining of UPR activation and p-tau in the hippocampus (A) and in the cortex (B) are shown. pIRE1 $\alpha$ (a-d) and pPERK (e-h) are markers for UPR activation, and AT8 (i-l) detects $p$-tau. During euthermia ( $a$, e and i) there are no pIRE1 $\alpha$-, pPERK-and AT8-positive cells, but during early (b, fand j) and late torpor (c, $\mathrm{g}$ and $\mathrm{k}$ ) there is abundant reactivity for these markers. During arousal the reactivity of all markers is dissipated ( $\mathrm{d}, \mathrm{h}$ and $\mathrm{I})$. Scale bar: $100 \mu \mathrm{m}$

non-specific side effect of hypothermia. Moreover, stressinduced activation of the UPR is severely impaired under hypothermic conditions (Figure 4; Supplementary Figures 3 and 4). We therefore explored the hypothesis that the UPR activation observed during torpor is induced during euthermia and subsequently consolidated by the hypothermic state. To test this, SK-N-SH cells were incubated for $20 \mathrm{~h}$ at 37 and $26^{\circ} \mathrm{C}$. BiP mRNA levels were used as a readout for UPR activation, as similar results were found for all UPR targets in the previous experiments. In line with our hypothesis, we demonstrate that if the UPR is activated upon metabolic stress for $20 \mathrm{~h}$ at $37^{\circ} \mathrm{C}$ it remains activated after $20 \mathrm{~h}$ at $26^{\circ} \mathrm{C}$, even if the metabolic stressor is removed. Interestingly, if the cells are subsequently moved to $37^{\circ} \mathrm{C}$ for another $20 \mathrm{~h}$ without a metabolic stressor, the BiP mRNA levels return to normal (Figure 5). If the metabolic stressor is not removed the UPR remains activated, as expected. These data demonstrate that if the UPR is activated during euthermia, this activation is consolidated during hypothermia, but that upon restoration of normal metabolic conditions at $37^{\circ} \mathrm{C}$ the UPR is switched off. These results further strengthen the hypothesis that the UPR is an adaptive 

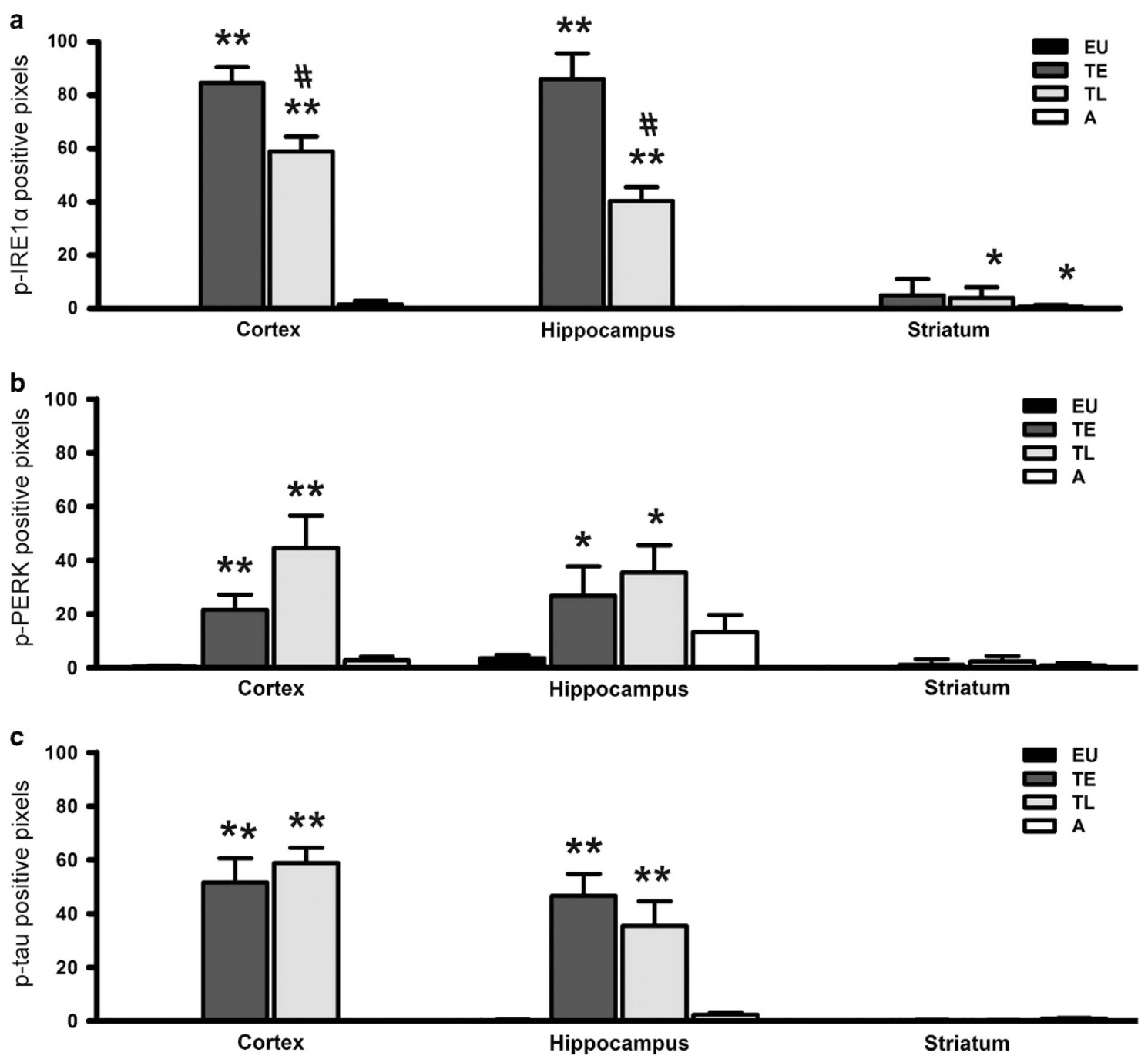

Figure 2 UPR and p-tau quantification of different brain areas during torpor. Coronal brain sections of wild-type Syrian hamsters were studied during euthermia, early torpor, late torpor and arousal. Images of the cortex, hippocampus and striatum of each animal were acquired with $\times 20$ objective and identical microscope settings. ImageJ software with threshold color plugin was used to quantify the images (see Materials and Methods for details). The positive pixels (mean \pm S.E.M. of $n=3-5$ animals per group) of pIRE1 $\alpha(\mathbf{a})$, pPERK (b) and AT8 (c) in cortex, hippocampus and striatum are shown. The quantification of positive reactivity demonstrate high levels of pIRE1 $\alpha$, pPERK and AT8 during early torpor (TE) and late torpor (TL) in cortex and hippocampus and almost no positive reactivity during euthermia (EU) and arousal (A) ( ${ }^{*} P<0.05$ relative to EU, ${ }^{* *} P<0.01$ relative to EU, ${ }^{\#} P<0.05$ TE relative to TL)

response that is actively induced under physiological hypometabolic conditions and actively reversed upon restoration of energy homeostasis.

Metabolic stress induces reversible p-tau via the UPR. Because UPR activation and tau phosphorylation are observed together not only under pathological but also under physiological conditions in the brain, it is likely that there is a direct functional connection between the two events. To test this, differentiated SK-N-SH cells were treated with the UPR inducer TM for $20 \mathrm{~h}$. The phosphorylation of tau at Ser396 is significantly increased in TM-treated cells (Figure 6a). Interestingly, when differentiated SK-N-SH cells were treated with 2DG for $20 \mathrm{~h}$ to induce similar metabolic stress that occurs during torpor, tau phosphorylation is significantly increased (Figure 6b). No change in the total tau levels were observed in TM- and 2DG-treated cells (Figure 6).
This demonstrates that tau phosphorylation is induced by UPR activation. In addition, the data show that metabolic stress is an activator of the UPR that leads to increased p-tau levels. In combination with our observations in the hypometabolic brain in vivo, these data suggest that tau phosphorylation is part of the UPR-mediated adaptive response to metabolic stress. To investigate whether tau phosphorylation is a normal physiological process that is reversible upon restoration of metabolic homeostasis, the UPR was activated in differentiated SK-N-SH cells for $20 \mathrm{~h}$ with 2DG. Subsequently the cells were incubated for another $20 \mathrm{~h}$ in the presence or absence of a metabolic stressor. The levels of $p$-tau are increased in the continuous presence of the metabolic stressor, as expected (Figure 7). However, if the metabolic stressor is removed and washed away, p-tau levels return to normal (Figure 7), like UPR activation (Figure 5). In support of an adaptive mechanism, 

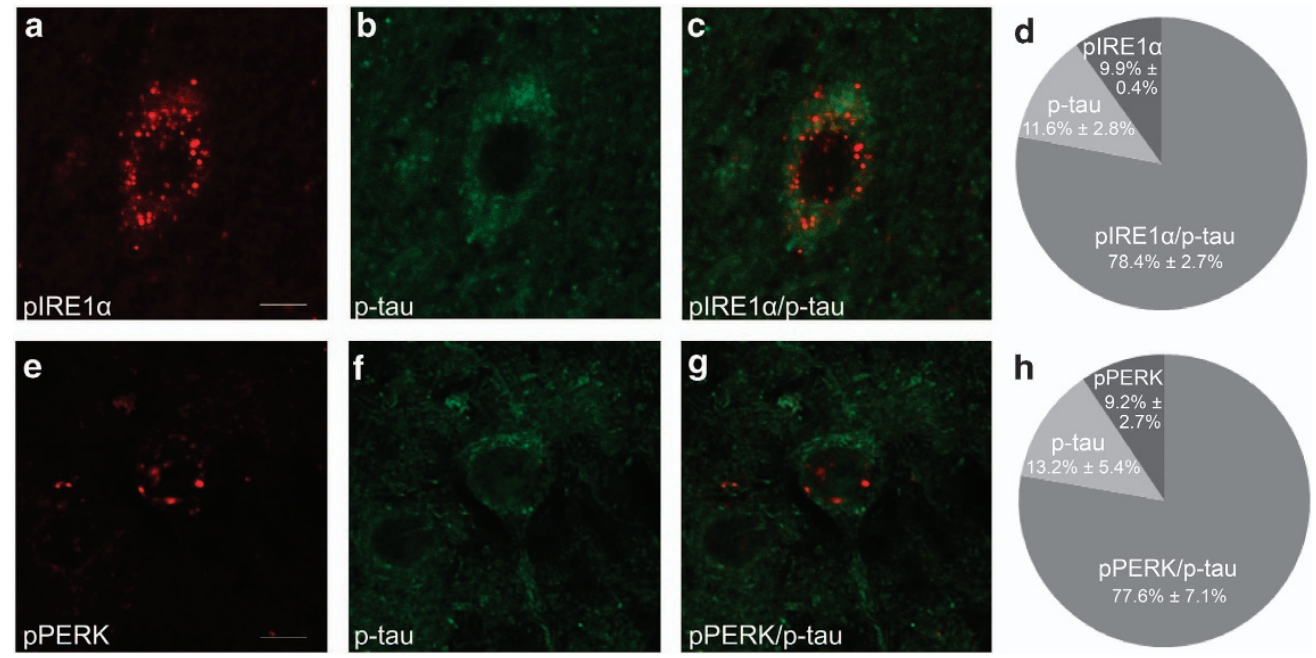

Figure 3 Spatial correlation between UPR activation and p-tau. Coronal brain sections of wild-type Syrian hamsters in early torpor and late torpor were analyzed by double-immunofluorescence staining of UPR markers (pIRE1 $\alpha$ and pPERK) and p-tau (AT8). Representative double-immunofluorescent images of early torpor obtained by confocal microscopy are shown. pIRE $1 \alpha(\mathbf{a})$ and pPERK (e) are depicted in red and AT8 (b and $\mathbf{f}$ ) in green. The overlays of the markers demonstrate colocalization of plRE1 $\alpha$ and AT8 (c) and colocalization of pPERK and AT8 (g) in the same neuron. Quantification of cells with positive reactivity (mean \pm S.E.M. of $n=120$ ) demonstrate that $>75 \%$ of the neurons show both UPR activation and p-tau (d and $\mathbf{h})$. Scale bar: $10 \mu \mathrm{m}$
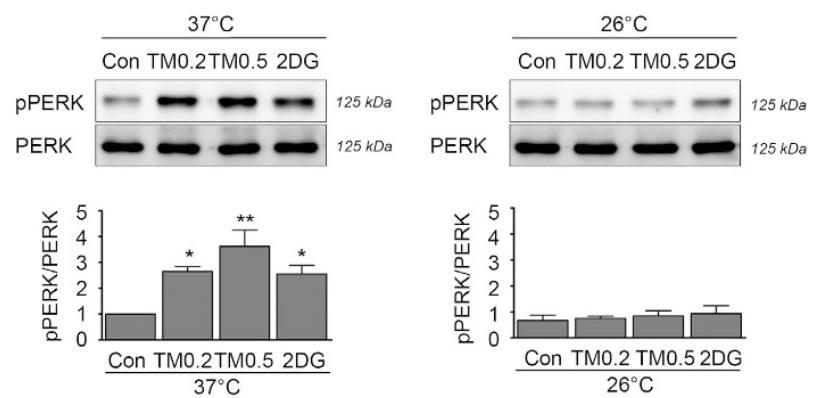

Figure 4 Hypothermia does not elevate pPERK levels. Differentiated SK-N-SH cells were incubated at 37 or $26^{\circ} \mathrm{C}$ for $20 \mathrm{~h}$ in the absence (Con) or presence of UPR inducers: 0.2 and $0.5 \mu \mathrm{g} / \mathrm{ml}$ TM and $40 \mathrm{mM}$ 2DG. Immunoprecipitation and western blot analyze of pPERK was performed. There is no induction of pPERK at $26^{\circ} \mathrm{C}$ in the untreated condition. An increase of pPERK is visible at $37^{\circ} \mathrm{C}$ after $20 \mathrm{~h}$ treatment with TM and 2DG. This elevation of pPERK after treatment is not observed at $26^{\circ} \mathrm{C}$. All data are presented as mean \pm S.D. of an experimental $n=3$ and normalized to the untreated $37^{\circ} \mathrm{C}$ control condition $\left({ }^{*} P<0.05,{ }^{* *} P<0.01\right)$

these data demonstrate that both UPR activation and tau phosphorylation are reversed upon restoration of metabolic homeostasis.

If the UPR is key to metabolic stress-induced tau phosphorylation, intervention in the UPR may provide an opportunity to prevent this early event in tau pathology. To inhibit the proteotoxic stress in the ER induced by 2DG, we employed the chemical chaperone tauroursodeoxycholic acid (TUDCA), which effectively inhibits overall UPR activation and thus of its downstream targets. ${ }^{23,24}$ To confirm the effect of TUDCA, phosphorylated elF2 $\alpha$ (p-elF $2 \alpha$ ), which is phosphorylated by activated PERK, was measured. Differentiated SK-N-SH cells were pre-treated with TUDCA for $1 \mathrm{~h}$ before the metabolic stressor 2DG was added to the medium for $20 \mathrm{~h}$. Indeed, TUDCA inhibits the 2DG-induced increase in p-elF2 $\alpha$ levels, as expected (Figure 8). Interestingly, this inhibition of the UPR also prevents 2DG-induced tau phosphorylation. This demonstrates not only that the increase in p-tau upon metabolic stress is mediated by the UPR, but also identifies the UPR as a putative target for therapeutic intervention. Recently, a selective inhibitor of the PERK pathway of the UPR has become available, GSK2606414. ${ }^{25}$ To test this small molecule, differentiated neuronal SK-N-SH cells were pretreated with the PERK inhibitor for $1 \mathrm{~h}$ before the metabolic stressor 2DG was added to the medium for $20 \mathrm{~h}$. Pretreatment with GSK2606414 inhibits the 2DG-induced increase in p-elF $2 \alpha$ levels, validating that it inhibits PERK activity in the human neuronal cell model (Figure 8). More importantly, inhibition of the PERK pathway by this compound also prevents the phosphorylation of tau upon treatment with 2DG. This implicates that intact UPR signaling is required for tau phosphorylation under metabolic stress and provides the first evidence that interference in the UPR is a possible therapeutic target in tauopathies.

\section{Discussion}

In the present study, we investigated whether UPR activation functionally connects metabolic dysfunction and tau phosphorylation. We demonstrate that metabolic stress activates the UPR resulting in increased tau phosphorylation. This adds to earlier data that show that thapsigargin treatment leads to tau phosphorylation. ${ }^{20,21}$ Thapsigargin induces the UPR via disruption of $\mathrm{ER} \mathrm{Ca}^{2+}$ homeostasis, however, it affects many different processes. Here, we show that two other inducers of the UPR - TM and 2DG - also result in tau phosphorylation. Moreover, we demonstrate involvement of the UPR using the chemical chaperone TUDCA and a selective inhibitor of the PERK pathway. A very interesting and striking observation is the concomitant reversibility of UPR activation and p-tau after return to the normal physiological state both in vitro and in vivo.

The p-tau observed in torpor is not aggregated and resembles the pre-tangle stages of tau pathology, in which we observe activation of the UPR in the human brain. ${ }^{15}$ 


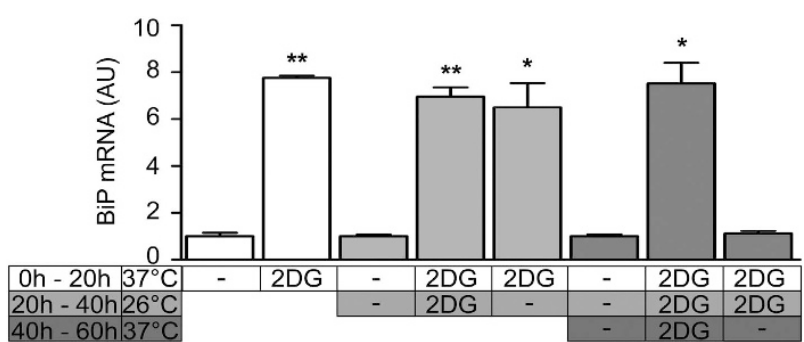

Figure 5 Activation of the UPR during euthermia is consolidated during hypothermia. Differentiated SK-N-SH cells were sequentially incubated at $37^{\circ} \mathrm{C}$ for $20 \mathrm{~h}$, at $26^{\circ} \mathrm{C}$ for $20 \mathrm{~h}$ and again at $37^{\circ} \mathrm{C}$ for $20 \mathrm{~h}$. Cells were treated in the absence or presence of $2 \mathrm{DG}$ to induce metabolic stress, and $2 \mathrm{DG}$ was added or removed at each subsequent temperature change as indicated. After each incubation period of $20 \mathrm{~h}, \mathrm{BiP}$ mRNA levels were determined by $\mathrm{qPCR} ; \mathrm{eEF} 2 \alpha$ was used as a reference gene. Data are presented as mean \pm S.D. in arbitrary units (AU) from triplicate observations of a representative experiment and are normalized to the untreated samples with the same incubation time. BiP mRNA levels are consolidated at $26^{\circ} \mathrm{C}$ in the absence of $2 \mathrm{DG}$ and are reversed upon restoration of metabolic homeostasis by removal of $2 D G$ at $37^{\circ} \mathrm{C}\left({ }^{\star} P<0.05,{ }^{\star \star} P<0.01\right)$
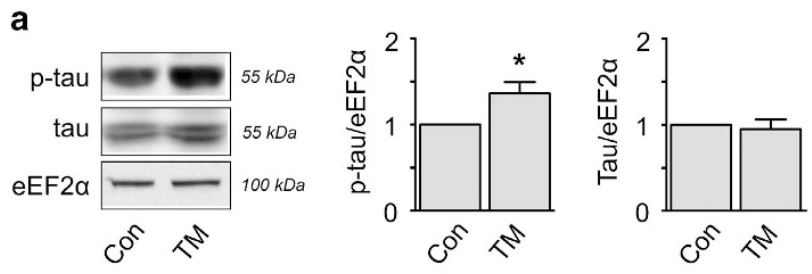

b
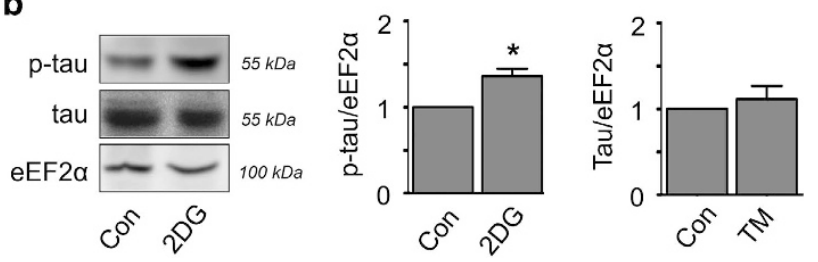

Figure 6 Metabolic stress functionally connects UPR activation and tau phosphorylation. Differentiated SK-N-SH cells were treated with TM (a) or 2DG (b) for $20 \mathrm{~h}$. Representative western blot of $n=3$ is shown. Tau Ser396 phosphorylation (p-tau) is increased after treatment with TM and 2DG. Total tau is not changed. Quantification of p-tau and tau is relative to loading control eEF2 $\alpha$. This experiment shows that UPR activation induces tau phosphorylation $\left({ }^{*} P<0.01\right)$

Reduction of the temperature below $37^{\circ} \mathrm{C}$ was previously shown to disturb the balance between tau kinase and phosphatase activity, and this has been suggested to cause increased tau phosphorylation as a result of a passive temperature-driven mechanism. ${ }^{22}$ In a recent and more extensive study, however, it was shown that the phosphorylation of tau is first actively induced as animals go into torpor and that this is passively consolidated by the lower temperature as a secondary event. ${ }^{5}$ Here, we demonstrate that in analogy to the torpor-induced phosphorylation of tau not hypothermia but hypometabolic stress is involved in the activation of the UPR, and that the UPR remains activated during the hypothermic state. Interestingly, in torpor UPR activation and p-tau followed a certain spatial hierarchy associated with energy metabolism. The cortex and hippocampus showed the most pronounced UPR activation and p-tau, whereas other areas such as the striatum showed almost no positive cells. These a

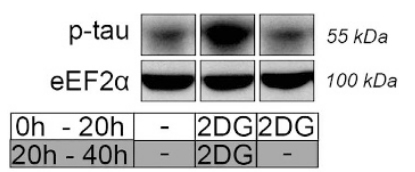

b

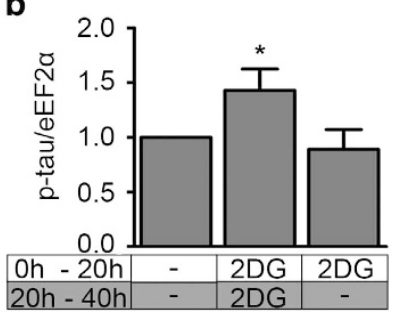

Figure 7 UPR activation and $p$-tau are reversed upon restoration of metabolic homeostasis. Differentiated SK-N-SH cells are treated with 2DG for $20 \mathrm{~h}$. Subsequently the cells were incubated for another $20 \mathrm{~h}$ with or without 2DG. Representative western blot (a) and quantification (b) of tau Ser396 phosphorylation ( $p$-tau) of $n=3$ is shown. After $40 \mathrm{~h}$ p-tau levels are increased after 2DG treatment, but are reversed if $2 D G$ is removed. This experiment demonstrates the reversibility of UPR-induced tau phosphorylation upon restoration of metabolic homeostasis $\left({ }^{\star} P<0.05\right)$
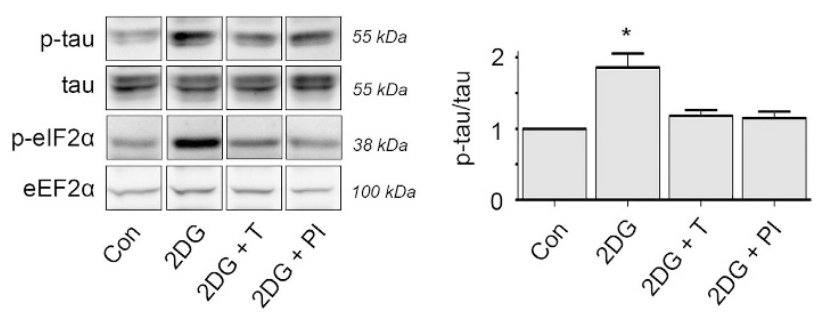

Figure 8 Inhibition of UPR prevents p-tau upon metabolic stress. Differentiated SK-N-SH cells were treated $20 \mathrm{~h}$ with 2DG alone or in the presence of TUDCA $(2 D G+T)$, a UPR inhibitor or a selective PERK inhibitor $(2 D G+P I)$. Representative western blot of $n=3$ is shown. Tau Ser396 phosphorylation ( $p$-tau) is significantly increased after treatment with 2DG, but is not significantly increased in presence of TUDCA or the PERK inhibitor. Quantification of $p$-tau is relative to total tau levels. P-elF2 $\alpha$, the first downstream target of activated PERK, is shown as an indicator of UPR activation. eEF2 $\alpha$ is used as a loading control. This experiment shows that $p$-tau can be prevented by inhibition of the UPR via the PERK pathway $\left({ }^{*} P<0.01\right)$

areas with high UPR activation and p-tau levels are areas that typically have a high metabolic demand, ${ }^{26}$ suggesting that metabolic stress is a critical factor. Interestingly, these metabolically demanding regions are also the brain areas that are affected early in AD. ${ }^{27}$

Metabolic stress has already been described as an important feature in AD. The majority of AD patients display abnormal glucose metabolism, ranging from glucose intolerance to insulin resistance and type 2 diabetes mellitus. ${ }^{28}$ A reduced glucose metabolic rate is already observed in patients that are genetically predisposed to develop AD before any pathological signs of the disease manifest. ${ }^{29,30}$ Moreover, glucose metabolism is also decreased in brains of patients with mild cognitive impairment, which is a prodromal stage of AD. ${ }^{31-34}$ Recent data show that in cognitively normal subjects with early signs of disturbed peripheral glucose metabolism, glucose utilization is impaired in brain areas affected in AD. ${ }^{35}$ This indicates that peripheral metabolic dysfunction is associated with hypometabolism in the brain that is not necessarily a downstream effect of neurodegeneration. It is interesting to note that in a recent GWAS study, GLIS3, a major factor in glucose metabolism, was associated with increased tau levels in the cerebrospinal fluid, an early biomarker for AD-related changes in the brain. ${ }^{36}$ 
We hypothesize that metabolic stress induces UPR activation and tau phosphorylation in the brain as a reversible neuroprotective response. In line with this, we find that return to normal physiological metabolic conditions reverses both activation of the UPR and phosphorylation of tau in vitro and in vivo. However, if the hypometabolic state persists due to fundamental metabolic problems, for example, because of insulin resistance as was demonstrated to occur in $A D$ hippocampus, ${ }^{37}$ this may eventually lead to irreversible tau aggregation. These tau aggregates can then in turn contribute to prolonged UPR activation by interfering with ER-associated degradation, as was recently shown to occur in a transgenic tau model with a very aggressive phenotype. ${ }^{38,39}$ This further underlines the risk of prolonged UPR activation and the importance of balanced regulation.

Our data add to accumulating evidence that puts the UPR in the frame as an early therapeutic target for neurodegenerative diseases. ${ }^{40,41}$ In fact, interference in the UPR by deletion of the gene encoding PERK, EIF2AK3, or by administration of a selective PERK inhibitor similar to the one used in this study to inhibit UPR-induced tau phosphorylation showed beneficial effects in animal models for neurodegeneration. ${ }^{42-44}$ These effects are mediated via rescue of the attenuation of protein synthesis. In addition, here we show that inhibition of PERK reduces UPR-induced tau phosphorylation, indicating that the PERK pathway is at least involved in UPR-mediated tau phosphorylation. Interestingly, a polymorphism in the EIF2AK3 gene was shown to be associated with risk to develop progressive supranuclear palsy (PSP), another neurodegenerative tauopathy. ${ }^{45}$ In brains of PSP patients, UPR activity is found in neurons with diffuse p-tau, as in AD. ${ }^{16}$ This underlines the importance of proper PERK function in the protection against tau pathology.

Although our data suggest that tau phosphorylation upon metabolic stress is mediated by the UPR, it is still elusive how the UPR affects the activity of tau kinases or phosphatases. For one of the major tau kinases, glycogen synthase kinase 3, it was demonstrated that the UPR increases the activity, ${ }^{21,46}$ but it is not known how this works mechanistically. UPR activation may also lead to downregulation of phosphatases, by inhibition of the activity via phosphorylation. As IRE1 and PERK are kinases with an increasing list of substrates, ${ }^{41}$ this could be a direct effect of these kinases or indirect via activation of a downstream substrate kinase. Finally, the levels of phosphatases or kinases may be regulated by activation of the transcriptional IRE1 and ATF 6 pathways of the UPR. Alternatively, the PERK pathway has drastic effects on the proteome via the overall translation inhibition and increased translation of selective mRNAs, the latter also leading to increased transcription of target genes of activating transcription factor 4 (ATF4). Future studies will be directed at elucidation of the signaling pathway that connects UPR activation to $p$-tau, to develop selective therapeutic intervention methods.

In conclusion, we present the first evidence for a connection between UPR activation and tau phosphorylation as a physiological metabolic stress mechanism not primarily associated with pathology. Importantly, the reversibility of both UPR activation and tau phosphorylation indicates that intervention in UPR regulation may not only prevent, but possibly even dissipate early tau accumulations in the tauopathy brain.

\section{Materials and Methods}

Syrian hamsters. This study was performed with wild-type Syrian hamsters (Mesocricetus auratus) derived from the breeding colony in Haren, University of Groningen. Hamsters were housed in Macrolon type 3 cages on sawdust bedding, with hay as nesting material. Water and food were available ad libitum throughout the experiment. Syrian hamsters were subjected to torpor conditions as previously described. ${ }^{19} \mathrm{~A}$ control group, the euthermic group (EU), was continuously housed at $21( \pm 1)$ degree and summer photoperiod ( $14 \mathrm{~h}$ of light and $10 \mathrm{~h}$ of darkness). Animals in the EU group were sampled in the inactive phase. Animals in the experimental group were killed at different time points during torpor: animals in early torpor (TE) were sampled after $24.1 \mathrm{~h}$ (S.E.M. $0.51 \mathrm{~h}$ ) of inactivity, animals in late torpor (TL) were sampled after $94.7 \mathrm{~h}$ (S.E.M. $2.93 \mathrm{~h}$ ) of inactivity and animals in the arousal group were sampled $2.5 \mathrm{~h}$ (S.E.M. $0.04 \mathrm{~h}$ ) after induction of arousal from torpor. Mouth temperatures of the animals, taken at the moment of killing, confirmed the state of the animals: TE: $6.6^{\circ} \mathrm{C}$ (S.E.M. $0.18^{\circ} \mathrm{C}$ ), TL: $6.8^{\circ} \mathrm{C}$ (S.E.M. $0.47^{\circ} \mathrm{C}$ ), arousal: $32.1^{\circ} \mathrm{C}$ (S.E.M. $0.8^{\circ} \mathrm{C}$ ) and EU: $35.4^{\circ} \mathrm{C}$ (S.E.M. $0.39^{\circ} \mathrm{C}$ ). Syrian hamsters were killed by intraperitoneal injection of $60 \mathrm{mg} / \mathrm{kg}$ pentobarbital. Subsequently brains were dissected and frozen in $-80^{\circ} \mathrm{C}$ for protein lysates or animals were transcardially perfused with $100 \mathrm{ml}$ phosphate-buffered saline (PBS) followed by $300 \mathrm{ml} 4 \%$ paraformaldehyde in $0.1 \mathrm{M}$ PBS. Brains were dissected, post-fixated for $24 \mathrm{~h}$ in $4 \%$ paraformaldehyde in $0.1 \mathrm{M} \mathrm{PBS}$ at $6{ }^{\circ} \mathrm{C}$, and stored in PBS with $1 \%$ sodium azide at $6{ }^{\circ} \mathrm{C}$ for later immunohistochemistry analysis. The experiments were conducted by following the principles of laboratory animal care and were approved by the Animal Experiments Committee of the University of Groningen (license number DEC-2954).

Cell culture, differentiation and treatment. SK-N-SH, a human neuroblastoma cell line (European Collection of Cell Cultures \#86012802, Salisbury, UK), was cultured in Dulbecco's modified Eagle's medium with GlutaMAX supplemented with $10 \%$ (v/v) fetal calf serum (Sigma, St Louis, MO, USA), $100 \mathrm{U} / \mathrm{ml}$ penicillin and $100 \mu \mathrm{g} / \mathrm{ml}$ streptomycin. Before treatment, cells were kept at $37{ }^{\circ} \mathrm{C}, 5 \% \mathrm{CO}_{2}$ and $95 \%$ humidity. SK-N-SH cells were differentiated with trans-retinoic acid (Sigma) at a final concentration of $10 \mu \mathrm{M}$ for 5-10 days. In general, to activate the UPR, cells were treated with 0.2 or $0.5 \mu \mathrm{g} / \mathrm{ml} \mathrm{TM}$ or with $40 \mathrm{mM} 2 \mathrm{DG}$ at $37^{\circ} \mathrm{C}$. To inhibit UPR activation, SK-N-SH cells were preincubated for $1 \mathrm{~h}$ with $5 \mathrm{mM}$ TUDCA or $0.3 \mu \mathrm{M}$ PERK inhibitor I, GSK2606414 (PI) (Millipore, Billerica, MA, USA). For the hypothermia experiments, cells were incubated between 26 and $37^{\circ} \mathrm{C}$ as indicated. All temperature conditions were normalized and compared with simultaneously incubated $37^{\circ} \mathrm{C}$ control condition. To test the reversibility of the UPR, cells were subsequently incubated for $20 \mathrm{~h}$ at 37,26 and $37^{\circ} \mathrm{C}$. The metabolic stressor $2 \mathrm{DG}$ was removed by washing the cells two times with regular medium.

Immunoprecipitation. SK-N-SH cells were plated and differentiated in 9-cm dishes. After treatment, cells were scraped in $500 \mu \mathrm{l}$ of lysis buffer (1\% Triton $\mathrm{X}-100,150 \mathrm{mM} \mathrm{NaCl}, 20 \mathrm{mM}$ Hepes pH7.5, 10\% glycerol, $1 \mathrm{mM}$ EDTA supplemented with protease inhibitors (complete protease inhibitors from Roche, Penzberg, Germany) and phosphatase inhibitors (PhosSTOP, Roche). The lysate (supernatant) was obtained after $5 \mathrm{~min}$ spin at $4^{\circ} \mathrm{C}$. Supernatant protein content was determined by the Bio-Rad Protein Assay (Bio-Rad, Hercules, CA, USA). Total lysate was precleared with $15 \mu$ l washed protein G-Sepharose for $60 \mathrm{~min}$ at room temperature. For immunoprecipitation $1 \mathrm{mg}$ lysate was incubated with $2 \mu \mathrm{g}$ PERK antibody linked to $15 \mu \mathrm{l}$ of packed volume of protein G-Sepharose under rotation at $4^{\circ} \mathrm{C}$ overnight. Proteins were washed three times with lysis buffer and once with PBS. Subsequently, proteins were boiled at $95^{\circ} \mathrm{C}$ for $5 \mathrm{~min}$ and supernatant was used for SDS-PAGE and western blotting of pPERK and PERK.

SDS-PAGE and western blotting. Cells were harvested by scraping in $1 \%$ Triton X-100 TBS lysis buffer supplemented with protease and phosphatase inhibitors. Cell lysates were vigorously mixed after $5 \mathrm{~min}$ incubation on ice and centrifuged for $5 \mathrm{~min}$ at $20000 \times g$ at $4^{\circ} \mathrm{C}$. Supernatant protein content was determined by the Bio-Rad Protein Assay (Bio-Rad). Equal amounts of protein were loaded on appropriate percentage polyacrylamide gels, $8 \%$ for BiP and $10 \%$ for the other proteins and blotted onto PVDF membrane (Millipore) using a semi-dry electroblotting apparatus. Blots were preincubated with $5 \%$ bovine serum 
Table 1 Primary antibodies

\begin{tabular}{|c|c|c|c|}
\hline Antibody & Species & Dilution & Company \\
\hline \multicolumn{4}{|c|}{ Antibodies for western blot analyses } \\
\hline $\mathrm{BiP} / \mathrm{GRP} 78$ & Goat & $1: 1000$ in $5 \%$ BSA/TBS-T & Santa-Cruz, Dallas, TX, USA \\
\hline $\mathrm{eEF} 2 \alpha$ & Rabbit & $1: 1000$ in $5 \%$ BSA/TBS-T & Cell Signaling, Danvers, MA, USA \\
\hline p-elF2 $\alpha$ & Rabbit & $1: 500$ in 5\% BSA/TBS-T & Abcam, Cambridge, UK \\
\hline PERK & Goat & $1: 500$ in $5 \%$ BSA/TBS & Santa-Cruz \\
\hline pPERK & Rabbit & $1: 500$ in $5 \%$ BSA/TBS & Santa-Cruz \\
\hline Total elF $2 \alpha$ & Rabbit & $1: 500$ in $5 \%$ BSA/TBS-T & Cell Signaling \\
\hline Tau & Rabbit & $1: 1000$ in $2.5 \%$ milk/PBS-T & DAKO \\
\hline p-tau $\left(\right.$ Ser $\left.^{396}\right)$ & Mouse & $1: 1000$ in $1 \%$ BSA/PBS-T & Cell Signaling \\
\hline \multicolumn{4}{|c|}{ Antibodies for immunohistochemistry } \\
\hline AT8 & Mouse & $1: 2000$ (single) & Pierce, Rockford, IL, USA \\
\hline AT8-BIO & Mouse & $1: 500$ (double) & Thermo scientific \\
\hline $\operatorname{pIRE} 1 \alpha$ & Rabbit & $1: 80.000$ (single), $1: 20.000$ (double) & Novus Biologicals, Littleton, CO, USA \\
\hline pPERK (Thr980) & Rabbit & $1: 4000$ (single), $1: 400$ (double) & Santa-Cruz \\
\hline
\end{tabular}

albumin (Boehringer, Mannheim, Germany) in TBS-T (0.05\% Tween-20 in TBS) for $60 \mathrm{~min}$ at room temperature and subsequently incubated at $4{ }^{\circ} \mathrm{C}$ overnight with primary antibodies. Membranes were washed $3 \times 10 \mathrm{~min}$ in TBS-T and subsequently incubated with species-specific secondary antibodies conjugated to horseradish peroxidase (dilution 1:2000, Dako, Glostrup, Denmark). Reactive protein bands were visualized using LumiLightPLUS Western blotting substrate (Roche Applied Science, Penzberg, Germany) and a LAS-3000 luminescent image analyzer (Fuji Photo Film (Europe), Kleve, Germany). Results were analyzed using Advanced Image Data Analyzer software (Raytest, Straubenhardt, Germany) version 3.44.035. The control and treated samples were all loaded on the same SDS-PAGE gel. The primary antibodies and their dilution factors are listed in Table 1.

RNA isolation, cDNA synthesis and real-time qPCR. Cells were lysed and scraped in TRIzol reagent (Invitrogen, Carlsbad, CA, USA) and organicand aqueous phase separation was initiated by the addition of and mixing with chloroform (Merck, Whitehouse Station, NJ, USA). Subsequently, RNA isolation was performed automated with an RNeasy MiniKit on a Qiacube (Qiagen, Venlo, the Netherlands; according to the manufacturer's protocol). RNA purity and integrity was assessed spectrophotometrically on a NanoDrop 2000 spectrophotometer (Thermo Scientific, Waltham, MA, USA). CDNA synthesis was performed on $0.5-1.0 \mu \mathrm{g}$ of RNA per reaction (RNA sample quantities do not vary within each experiment) using a SuperScript II Reverse Transcriptase Kit (Invitrogen). Priming of mRNA poly-A tails was performed with 125 pmol oligo(dT) $)_{12}-\mathrm{VN}$ primer in a final volume of $10 \mu \mathrm{l}$ and was incubated at $70^{\circ} \mathrm{C}$ for $10 \mathrm{~min}$. $\mathrm{MgCl}_{2}(2 \mathrm{mM})$, dNTPs $(0.5 \mathrm{mM}), 5 \mu \mathrm{l} 5 \times$ First-Strand Buffer and $100 \mathrm{U}$ SuperScript II reverse transcriptase were added to the primed mRNAs in a final volume of $25 \mu \mathrm{l}$ per reaction and incubated at $42^{\circ} \mathrm{C}$ for $60 \mathrm{~min}$. The reverse transcription reaction was stopped by incubation at $70^{\circ} \mathrm{C}$ for $10 \mathrm{~min}$. Per sample $1 \mu \mathrm{l}$ cDNA was pipetted in triplicate into a 384-well plate and dried in a DNA110 SpeedVac (Thermo Scientific). Primers and probe combinations are provided in Table 2.

Immunohistochemistry. Prior to cutting, brains were cryoprotected by submergence in $30 \%$ sucrose and $1 \%$ sodium azide in $0.1 \mathrm{M} \mathrm{PBS}$ at $6{ }^{\circ} \mathrm{C}$ for $48 \mathrm{~h}$. Brains were cut into $30 \mu \mathrm{m}$ coronal sections. The sections were immersed in $0.3 \%$ $\mathrm{H}_{2} \mathrm{O}_{2}$ in Tris-buffered saline (TBS) for $30 \mathrm{~min}$ to quench endogenous peroxidase activity. Sections were treated with $10 \mathrm{mmol} / \mathrm{l}, \mathrm{pH} \mathrm{6.0}$, sodium citrate buffer for $10 \mathrm{~min}$ at $99^{\circ} \mathrm{C}$ for antigen retrieval (except for the pPERK antibody) and subsequently incubated with primary antibodies at room temperature overnight. Antibodies (Table 1) were diluted in TBS containing $0.5 \%$ Triton X-100. Negative controls for all immunostainings were generated by omission of primary antibodies. Sections were washed with TBS. For the single staining, the sections were subsequently incubated for 120 min with undiluted EnVision/HRP anti-rabbit/mouse (Dako, Hamburg, Germany). Color was developed using 3, $3^{\prime}$-diaminobenzidine (EnVision detection system/HRP 1:50, DakoCytomation, Glostrup, Denmark) as a chromogen. After fixing sections on glass slides they were counterstained with hematoxylin and mounted using Depex (BDH Laboratories Supplies, East Grinstead, UK). For the double-immunofluorescence staining, the sections were incubated with either pPERK or pIRE1 $\alpha$ at room
Table 2 Primers and probes used for $\mathrm{qPCR}$

\begin{tabular}{llcc}
\hline $\begin{array}{l}\text { Target } \\
\text { gene }\end{array}$ & Sequence $\mathbf{5}^{\prime} \mathbf{- 3}^{\prime}$ & $\begin{array}{c}\text { Universal } \\
\text { Probe \# }\end{array}$ & $\begin{array}{c}\text { Product } \\
\text { length (bp) }\end{array}$ \\
\hline $\mathrm{BiP}$ & $\begin{array}{l}\text { fw: catcaagttcttgccgttca } \\
\text { rev: tcttcaggagcaatgtcttgt } \\
\text { fw: aaggcactgagcgtatcatgt }\end{array}$ & 10 & 99 \\
CHOP & 21 & 105 \\
GAPDH & $\begin{array}{l}\text { fw: tccaagatacacttccttcttgaaca } \\
\text { rev: ggcagagattcacc } \\
\text { fw: caatggacaaatctcctttt } \\
\text { rev: aacctcatctctattaaaaacaccaaa }\end{array}$ & 45 & 78 \\
eEF2 & 63 & 122 \\
\hline
\end{tabular}

Probe numbers refer to numbers in the Roche universal probe library

temperature overnight and subsequently incubated for $120 \mathrm{~min}$ with undiluted EnVision/HRP anti-rabbit (Dako). Color was developed using rhodamine tyramine $\left(1: 3000,0.03 \% \mathrm{H}_{2} \mathrm{O}_{2}, 5 \mathrm{~min}\right)$, which was made as previously described. ${ }^{47}$ Afterwards sections were incubated overnight with AT8-BIO (Thermo Scientific) and streptavidin ALEXA488 (1:500, Life Technologies, Carlsbad, CA, USA) for 120 min. Finally, the sections were mounted using vectashield.

Immunohistochemical images were obtained on an Olympus BX41 (Olympus, Tokyo, Japan) using cell $\mathrm{D}$ software (Olympus). For quantification of UPR activation and $\mathrm{p}$-tau, a representative area in the cortex, hippocampus and striatum of each animal was acquired with $\times 20$ objective. The same microscope settings were used for all animals. ImageJ software with threshold color plugin was used to quantify the area of interest that was immunoreactive for pIRE1 $\alpha$, pPERK and AT8 antibody. An area with positive reactivity was selected to determine the threshold. The threshold was the same for all brain areas and torpor stages. Each image was converted to an 8-bit binary image to measure the surface of positive pixels. The spatial correlation of pIRE1 $\alpha$, pPERK and AT8 was analyzed on a Leica TCS-SP2 confocal microscope (Leica, Wetzlar, Germany). For quantification, 40 cells with positive reactivity for one of the antibodies were analyzed three times. The percentage of cells positive for pIRE $1 \alpha$, pPERK or AT8 and the percentage of cells positive for both pPERK and AT8 or pIRE $1 \alpha$ and AT8 was calculated.

Statistical analysis. GraphPad Prism software was used for graphs and statistical analysis. The qPCR data are presented as mean \pm S.D., the other experiments are presented as mean \pm S.E.M. Data represent independent experiments of $n=3$ or more. All data are normalized and compared with the untreated $37^{\circ} \mathrm{C}$ control sample. For multiple statistical comparisons ANOVA, Tukey test was used. Two-sided unpaired Student's $t$-test was used for single statistical comparison. No significance difference was defined as $P>0.05$.

\section{Conflict of Interest}

The authors declare no conflict of interest. 
Acknowledgements. This study was supported by the Internationale Stichting Alzheimer Onderzoek Nederland (ISAO \#10502) to WS and an AMC $\mathrm{PhD}$ fellowship to $\mathrm{JMvdH}$. We thank Frank Baas and Piet Eikelenboom for stimulating discussions.

1. Ballatore C, Lee VM, Trojanowski JQ. Tau-mediated neurodegeneration in Alzheimer's disease and related disorders. Nat Rev Neurosci 2007; 8: 663-672.

2. Dixit R, Ross JL, Goldman YE, Holzbaur EL. Differential regulation of dynein and kinesin motor proteins by tau. Science 2008; 319: 1086-1089.

3. Lee HG, Perry G, Moreira PI, Garrett MR, Liu Q, Zhu X et al. Tau phosphorylation in Alzheimer's disease: pathogen or protector? Trends Mol Med 2005; 11: 164-169.

4. Morin P Jr., Storey KB. Mammalian hibernation: differential gene expression and novel application of epigenetic controls. Int J Dev Biol 2009; 53: 433-442.

5. Stieler JT, Bullmann T, Kohl F, Toien O, Bruckner MK, Hartig W et al. The physiological link between metabolic rate depression and tau phosphorylation in mammalian hibernation. PLoS One 2011; 6: e14530

6. Dausmann KH, Glos J, Ganzhorn JU, Heldmaier G. Physiology: hibernation in a tropical primate. Nature 2004; 429: 825-826.

7. Yanagisawa M, Planel E, Ishiguro K, Fujita SC. Starvation induces tau hyperphosphorylation in mouse brain: implications for Alzheimer's disease. FEBS Lett 1999; 461: 329-333.

8. Kim B, Backus C, Oh S, Hayes JM, Feldman EL. Increased tau phosphorylation and cleavage in mouse models of type 1 and type 2 diabetes. Endocrinology 2009; 150: 5294-5301.

9. Planel E, Tatebayashi Y, Miyasaka T, Liu L, Wang L, Herman M et al. Insulin dysfunction induces in vivo tau hyperphosphorylation through distinct mechanisms. J Neurosci 2007; 27: $13635-13648$

10. Ke YD, Delerue F, Gladbach A, Gotz J, Ittner LM. Experimental diabetes mellitus exacerbates tau pathology in a transgenic mouse model of Alzheimer's disease. PLoS One 2009; 4: e7917.

11. Hoglinger GU, Lannuzel A, Khondiker ME, Michel PP, Duyckaerts C, Feger J et al. The mitochondrial complex I inhibitor rotenone triggers a cerebral tauopathy. J Neurochem 2005; 95: 930-939.

12. Frisardi V, Solfrizzi V, Seripa D, Capurso C, Santamato A, Sancarlo D et al Metabolic-cognitive syndrome: a cross-talk between metabolic syndrome and Alzheimer's disease. Ageing Res Rev 2010; 9: 399-417.

13. Hoozemans JJ, Veerhuis R, van Haastert ES, Rozemuller JM, Baas F, Eikelenboom P et al. The unfolded protein response is activated in Alzheimer's disease. Acta Neuropathol 2005; 110: 165-172.

14. Unterberger U, Hoftberger R, Gelpi E, Flicker H, Budka H, Voigtlander T. Endoplasmic reticulum stress features are prominent in Alzheimer disease but not in prion diseases in vivo. J Neuropathol Exp Neurol 2006; 65: 348-357.

15. Hoozemans JJ, van Haastert ES, Nijholt DA, Rozemuller AJ, Eikelenboom P, Scheper W. The unfolded protein response is activated in pretangle neurons in Alzheimer's disease hippocampus. Am J Pathol 2009; 174: 1241-1251.

16. Nijholt DA, van Haastert ES, Rozemuller AJ, Scheper W, Hoozemans JJ. The unfolded protein response is associated with early tau pathology in the hippocampus of tauopathies. J Pathol 2012; 226: 693-702.

17. Walter $\mathrm{P}$, Ron $\mathrm{D}$. The unfolded protein response: from stress pathway to homeostatic regulation. Science 2011; 334: 1081-1086

18. Hoozemans JJ, Scheper W. Endoplasmic reticulum: the unfolded protein response is tangled in neurodegeneration. Int J Biochem Cell Biol 2012; 44: 1295-1298.

19. Hartig W, Stieler J, Boerema AS, Wolf J, Schmidt U, Weissfuss J et al. Hibernation model of tau phosphorylation in hamsters: selective vulnerability of cholinergic basal forebrain neurons-implications for Alzheimer's disease. Eur J Neurosci 2007; 25: 69-80.

20. Fu ZQ, Yang Y, Song J, Jiang Q, Lin ZC, Wang $Q$ et al. LiCl attenuates thapsigargininduced tau hyperphosphorylation by inhibiting GSK-3beta in vivo and in vitro. J Alzheimers Dis 2010; 21: 1107-1117.

21. Song L, De SP, Jope RS. Central role of glycogen synthase kinase-3beta in endoplasmic reticulum stress-induced caspase-3 activation. J Biol Chem 2002; 277: 44701-44708.

22. Planel E, Miyasaka T, Launey T, Chui DH, Tanemura K, Sato $\mathrm{S}$ et al. Alterations in glucose metabolism induce hypothermia leading to tau hyperphosphorylation through differential inhibition of kinase and phosphatase activities: implications for Alzheimer's disease. $J$ Neurosci 2004; 24: 2401-2411.

23. Ozcan U, Yilmaz E, Ozcan L, Furuhashi M, Vaillancourt E, Smith RO et al. Chemical chaperones reduce ER stress and restore glucose homeostasis in a mouse model of type 2 diabetes. Science 2006; 313: 1137-1140.

24. Xie Q, Khaoustov VI, Chung CC, Sohn J, Krishnan B, Lewis DE et al. Effect of tauroursodeoxycholic acid on endoplasmic reticulum stress-induced caspase-12 activation. Hepatology 2002; 36: 592-601.

25. Axten JM, Medina JR, Feng Y, Shu A, Romeril SP, Grant SW et al. Discovery of 7-methyl5-(1-\{[3-(trifluoromethyl)phenyl]acetyl\}-2,3-dihydro-1H-indol-5-yl)-7H-p yrrolo[2,3-d]pyrimidin4-amine (GSK2606414), a potent and selective first-in-class inhibitor of protein kinase $\mathrm{R}$ (PKR)-like endoplasmic reticulum kinase (PERK). J Med Chem 2012; 55: 7193-7207.
26. Bero AW, Yan P, Roh JH, Cirrito JR, Stewart FR, Raichle ME et al. Neuronal activity regulates the regional vulnerability to amyloid-beta deposition. Nat Neurosci 2011; 14: 750-756.

27. Braak H, Braak E. Staging of Alzheimer's disease-related neurofibrillary changes. Neurobiol Aging 1995; 16: 271-278.

28. Janson J, Laedtke T, Parisi JE, O'Brien P, Petersen RC, Butler PC. Increased risk of type 2 diabetes in Alzheimer disease. Diabetes 2004; 53: 474-481.

29. Small GW, Ercoli LM, Silverman DH, Huang SC, Komo S, Bookheimer SY et al. Cerebral metabolic and cognitive decline in persons at genetic risk for Alzheimer's disease. Proc Natl Acad Sci USA 2000; 97: 6037-6042.

30. Bateman RJ, Xiong C, Benzinger TL, Fagan AM, Goate A, Fox NC et al. Clinical and biomarker changes in dominantly inherited Alzheimer's disease. N Engl J Med 2012; 367: 795-804

31. Ishii H, Ishikawa H, Meguro K, Tashiro M, Yamaguchi S. Decreased cortical glucose metabolism in converters from CDR 0.5 to Alzheimer's disease in a community: the Osaki-Tajiri Project. Int Psychogeriatr 2009; 21: 148-156.

32. Drzezga A, Lautenschlager N, Siebner H, Riemenschneider M, Willoch F, Minoshima S et al. Cerebral metabolic changes accompanying conversion of mild cognitive impairment into Alzheimer's disease: a PET follow-up study. Eur J Nucl Med Mol Imaging 2003; 30: 1104-1113.

33. Mosconi L, Andrews RD, Matthews DC. Comparing brain amyloid deposition, glucose metabolism, and atrophy in mild cognitive impairment with and without a family history of dementia. J Alzheimers Dis 2013; 35: 509-524.

34. Nestor PJ, Fryer TD, Smielewski P, Hodges JR. Limbic hypometabolism in Alzheimer's disease and mild cognitive impairment. Ann Neurol 2003; 54: 343-351.

35. Burns CM, Chen K, Kaszniak AW, Lee W, Alexander GE, Bandy D et al. Higher serum glucose levels are associated with cerebral hypometabolism in Alzheimer regions. Neurology 2013; 80: 1557-1564.

36. Cruchaga C, Kauwe JS, Harari O, Jin SC, Cai Y, Karch CM et al. GWAS of cerebrospinal fluid tau levels identifies risk variants for Alzheimer's disease. Neuron 2013; 78: 256-268.

37. Talbot K, Wang HY, Kazi H, Han LY, Bakshi KP, Stucky A et al. Demonstrated brain insulin resistance in Alzheimer's disease patients is associated with IGF-1 resistance, IRS-1 dysregulation, and cognitive decline. J Clin Invest 2012; 122: 1316-1338.

38. Abisambra JF, Jinwal UK, Blair LJ, O'Leary JC III, Li Q, Brady S et al. Tau accumulation activates the unfolded protein response by impairing endoplasmic reticulum-associated degradation. J Neurosci 2013; 33: 9498-9507.

39. Stoveken BJ. Tau pathology as a cause and consequence of the UPR. J Neurosci 2013; 33: $14285-14287$.

40. Roussel BD, Kruppa AJ, Miranda E, Crowther DC, Lomas DA, Marciniak SJ. Endoplasmic reticulum dysfunction in neurological disease. Lancet Neurol 2013; 12: 105-118.

41. Scheper W, Hoozemans JJ. A new PERKspective on neurodegeneration. Sci Transl Med 2013; 5: 206fs37.

42. Ma T, Trinh MA, Wexler AJ, Bourbon C, Gatti E, Pierre P et al. Suppression of elF2alpha kinases alleviates Alzheimer's disease-related plasticity and memory deficits. Nat Neurosci 2013; 16: 1299-1305.

43. Moreno JA, Halliday M, Molloy $\mathrm{C}$, Radford H, Verity N, Axten JM et al. Oral treatment targeting the unfolded protein response prevents neurodegeneration and clinical disease in prion-infected mice. Sci Transl Med 2013; 5: 206ra138.

44. Moreno JA, Radford H, Peretti D, Steinert JR, Verity N, Martin MG et al. Sustained translational repression by elF2alpha-P mediates prion neurodegeneration. Nature 2012; 485: $507-511$.

45. Hoglinger GU, Melhem NM, Dickson DW, Sleiman PM, Wang LS, Klei L et al. Identification of common variants influencing risk of the tauopathy progressive supranuclear palsy. Nat Genet 2011; 43: 699-705.

46. Nijholt DA, Nolle A, van Haastert ES, Edelijn H, Toonen RF, Hoozemans JJ et al. Unfolded protein response activates glycogen synthase kinase-3 via selective lysosomal degradation. Neurobiol Aging 2013; 34: 1759-1771.

47. Hopman AH, Ramaekers FC, Speel EJ. Rapid synthesis of biotin-, digoxigenin-, trinitrophenyl-, and fluorochrome-labeled tyramides and their application for In situ hybridization using CARD amplification. J Histochem Cytochem 1998; 46: 771-777.

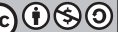

Cell Death and Disease is an open-access journal published by Nature Publishing Group. This work is licensed under a Creative Commons Attribution-NonCommercialShareAlike 3.0 Unported License. The images or other third party material in this article are included in the article's Creative Commons license, unless indicated otherwise in the credit line; if the material is not included under the Creative Commons license, users will need to obtain permission from the license holder to reproduce the material. To view a copy of this license, visit http://creativecommons.org/licenses/ by-nc-sa/3.0/ 